\title{
Seawater Magnetohydrodynamics Power Generator I Hydrogen Generator
}

\author{
Minoru TAKEDA \\ Graduate School of Maritime Sciences, Kobe University, Kobe 658-0022, Japan \\ takeda@maritime.kobe-u.ac.jp
}

Keywords: MHD generator, Superconducting technology, Helical flow, Seawater, Hydrogen

\begin{abstract}
A seawater magnetohydrodynamics (MHD) power generator / hydrogen generator is expected to become popular with the development of superconducting technology because of low loss and high efficiency. We have designed a new helical-type seawater MHD generator using a solenoid superconducting magnet, by considering the experimental results for a helical-type MHD ship. The experimental and computational results for the helical-type generator including the results of a recent study on hydraulic characteristics are discussed.
\end{abstract}

\section{Introduction}

A seawater magnetohydrodynamics (MHD) power generator / hydrogen generator is a unique system that not only directly transforms the kinetic energy of an ocean current / tidal current into electric energy but also generates hydrogen gas as a by-product. The energy of the ocean current / tidal current is expected to be effective as a sustainable energy source because of its independence of both weather and season in comparison with solar energy and wind power energy. It is great importance for an oceanic country such as Japan to develop the seawater MHD generator based on the sustainable ocean current / tidal current energy. In our work, experimental and computational studies on a seawater MHD generator using a superconducting magnet have been performed to investigate the application of superconductivity to maritime sciences. So far, a linear-type seawater MHD generator with a dipole superconducting magnet was constructed and experiments on power generation were successfully accomplished [1].

In seawater MHD generation, the applied magnetic field is an important factor determining the generator output and efficiency. A linear-type generator has the problem of requiring a large and strong superconducting magnet. To solve this problem, we designed a new helical-type seawater MHD generator using a solenoid superconducting magnet, by considering the experimental results for a helical-type MHD ship [2]. Preliminary experiments on generator output using the helical-type generator were carried out in a magnetic field of $7 \mathrm{~T}$ [3]. A numerical simulation was carried out continuously using a three-dimensional model [4], assuming its size to be equal to that of the generator. In this paper, experimental and computational studies on the helical-type seawater MHD power generator / hydrogen generator including our recent study on its hydraulic characteristics $[5,6]$ are reported.

\section{Preliminary Study}

Principle of Helical-Type MHD Generator. Fig. 1 shows the principle of the helical-type MHD generator. The helical-type generator consists of double-cylindrical coaxial electrodes, a helical insulation wall (a helical partition board) and a solenoid superconducting magnet. When seawater rotates around an anode in the presence of a magnetic field $B$ parallel to the coaxial direction, an electromotive force $V_{\mathrm{e}}$ is generated in accordance with the law of electromagnetic induction. $V_{\mathrm{e}}$ is proportional to $B$, the flow velocity $U$, the distance between the electrodes $D$ and $\sin \theta$, where $\theta$ is the 

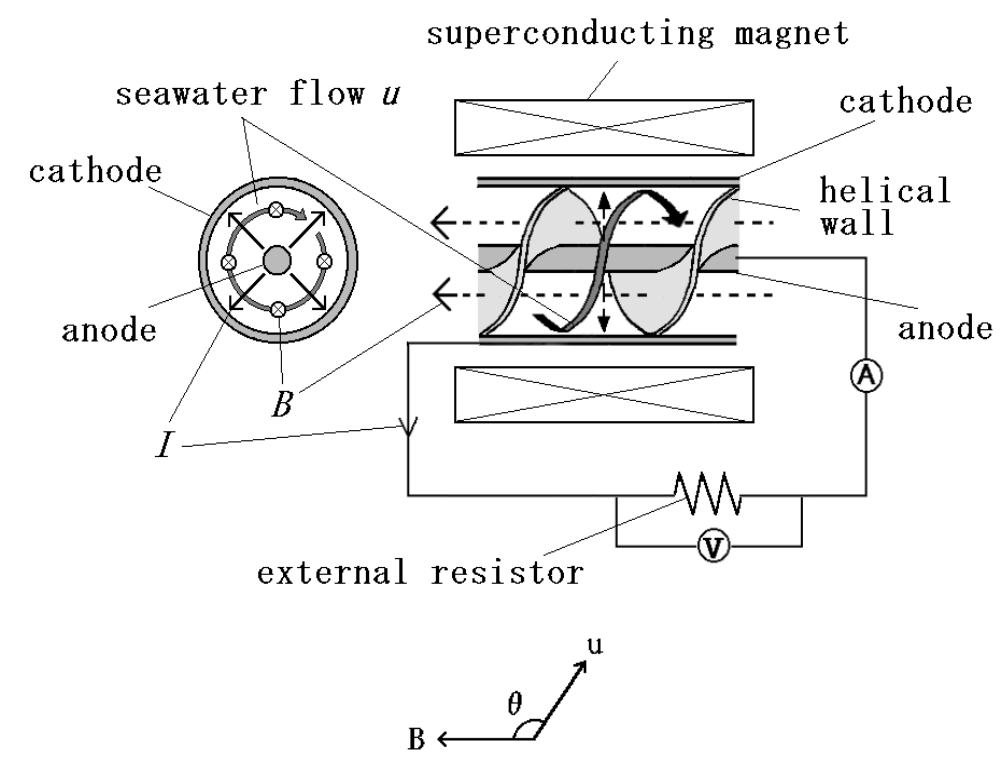

Fig. 1 Principle of the helical-type seawater MHD generator.

angle between the directions of $B$ and $U$. When $V_{\mathrm{e}}$ exceeds the electrolysis voltage $V_{\mathrm{d}}$, electric power $P$ and also hydrogen gas as a by-product are generated by the electric current $I$.

Experimental Apparatus. Fig. 2 shows a schematic diagram of the helical-type MHD generator [3]. The helical wall is made of polyvinyl chloride and has a spiral shape with a rotation number of 2.5 and a length of $140 \mathrm{~mm}$. The anode is a cylindrical rod $10 \mathrm{~mm}$ in diameter and $1350 \mathrm{~mm}$ long. The cathode is a cylindrical pipe $100 \mathrm{~mm}$ in outer diameter, $1.5 \mathrm{~mm}$ in thickness and $260 \mathrm{~mm}$ long. The electrodes are made of SUS316, which is nonmagnetic and corrosion-resistant. Fig. 3 shows a schematic diagram of the experimental system for the MHD generator. The system mainly consists of the generator, a cryostat with a $7 \mathrm{~T}$ solenoid superconducting magnet, a seawater tank, a seawater circulation pump, a flow meter, a pressure gauge and a thermometer.

Experimental Results. Experiments on the electromotive force and generator output were carried out to elucidate the fundamental characteristics of the helical-type generator using $\mathrm{NaCl}$ aqueous solution (3.4\%) [3]. The electromotive force was measured at various sample flows from 0 to $45 \mathrm{~m}^{3} / \mathrm{h}$ and magnetic fields from 0 to $7 \mathrm{~T}$. The generator output was also measured similarly using an external load of $1 \Omega$.

Fig. 4 represents the dependence of the electromotive force on the average flow velocity in a magnetic field of $7 \mathrm{~T}$. The electromotive force increased linearly with increasing average flow

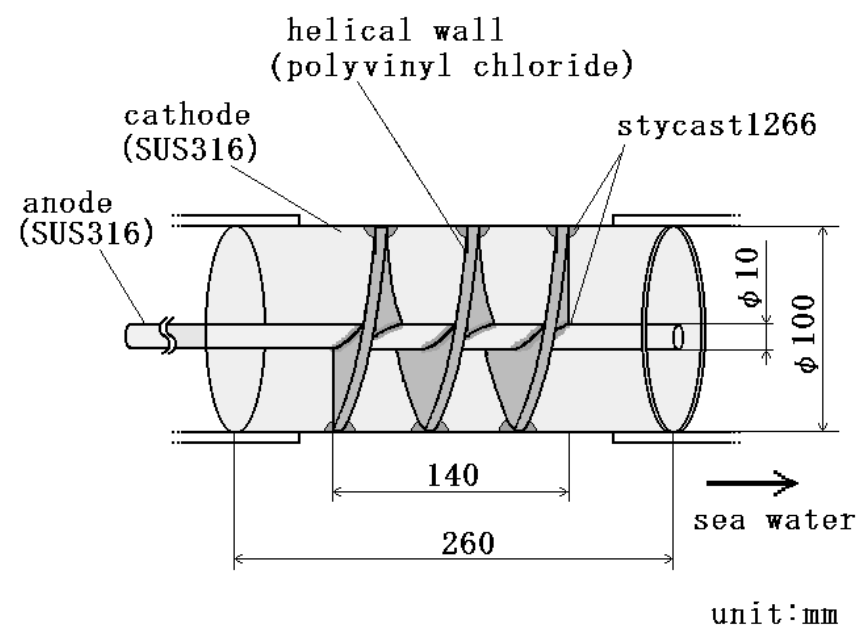

Fig. 2 Schematic diagram of the helical-type seawater MHD generator. 


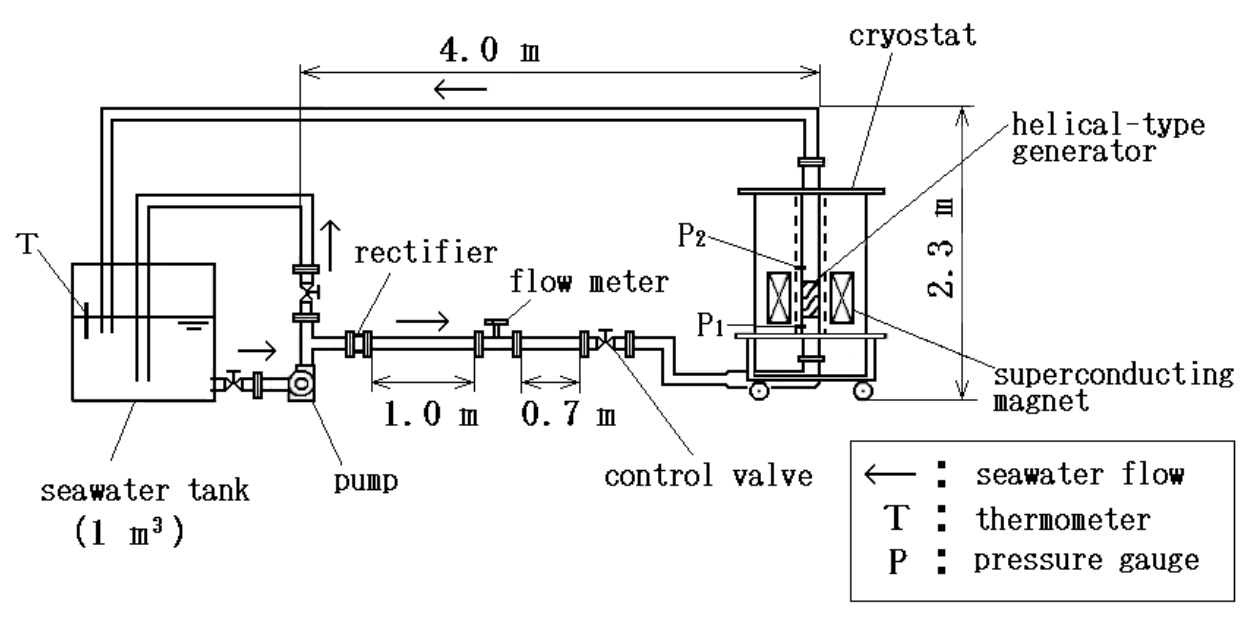

Fig. 3 Schematic diagram of the experimental setup.

velocity in a constant magnetic field. The dependence of the generator output on the average flow velocity in a magnetic field of $7 \mathrm{~T}$ is shown in Fig. 5. The generator output increased quadratically to average flow velocity over certain points. When the average flow velocity was kept at the maximum value $(5.6 \mathrm{~m} / \mathrm{s})$, a generator output of $0.05 \mathrm{~W}$ was attained. In this experiment, the electromotive force and generator output were small owing to the large flow loss of the generator.

\section{Numerical Simulation}

Simulation Model. To simulate the MHD generation system, we set up a three-dimensional model [4] and assumed its size to be equal to that of the generator used in the preliminary experiment. The simulation was carried out by the finite element method (FEM) on ANSYS multiphysics software (ANSYS Inc., version 8.0), which can perform a combined structural, thermal, fluid and electromagnetic simulation. The simulation includes two parts: fluid numerical analysis and electromagnetic numerical analysis.

Fluid Numerical Analysis. In this analysis, the values of flow loss, i.e., the pressure drop $\Delta P$ between the entrance and exit of the helical flow obtained in the experiment, were used. To confirm the validity of the numerical analysis of the generation system, we compared the flow rates $Q$ obtained experimentally with the numerical calculation for various pressure drops. The results were in good agreement for all pressure drops. Fig. 6 shows an example of the flow velocity distribution in the generation system at a flow rate of $45 \mathrm{~m}^{3} / \mathrm{h}$. In this figure, because a large number of nodes were used in the analysis, the vectors at selected nodes are distinguished by length as well as color. In addition,

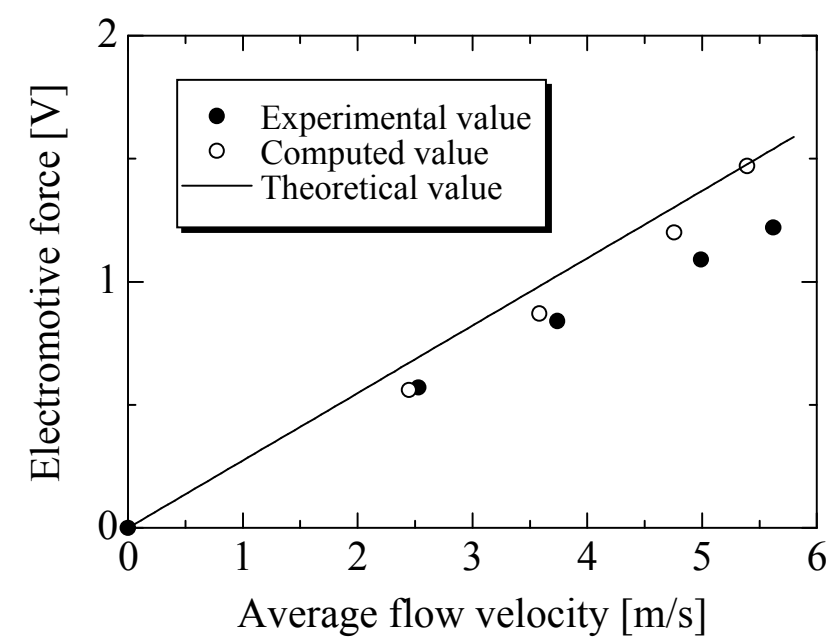

Fig. 4 Relationship between electromotive force and average flow velocity with $B=7 \mathrm{~T}$. 


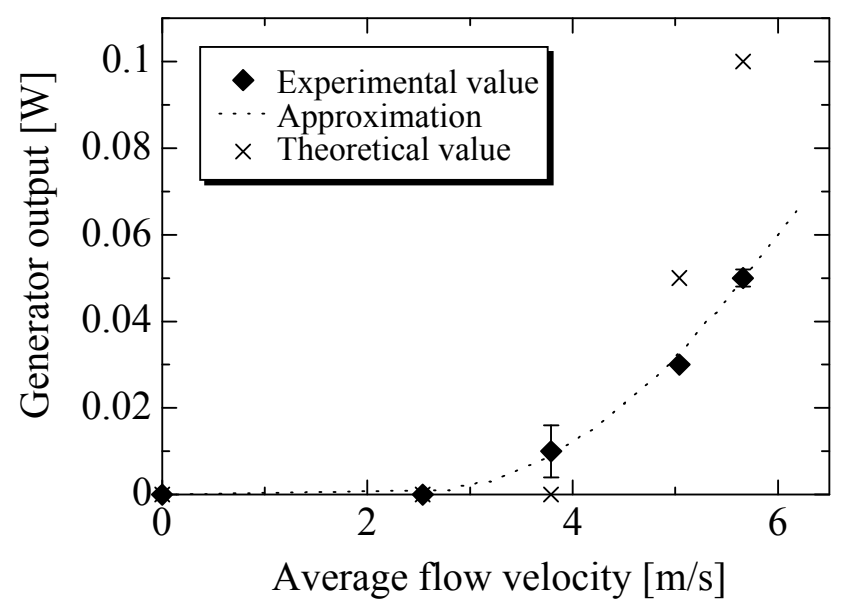

Fig. 5 Relationship between generator output and average flow velocity with $B=7 \mathrm{~T}$. Dotted line shows an approximation of experimental values.

to illustrate the directions of the velocity vectors clearly, a few of them are shown as thick black lines on the right side in Fig. 6.

Electromagnetic Numerical Analysis. The electromagnetic numerical analysis was carried out after the flow velocity distribution was obtained from the fluid numerical analysis. The helical wall acts as an insulator, and the magnetic permeability of each part in the generator was assumed to be equal to the value $\mu_{0}$ in a vacuum. The magnetic field was assumed to be homogeneous, and this analysis was performed by a technique of harmonic magnetic field analysis in ANSYS. Fig. 7 shows the electric current distribution at a flow rate of $45 \mathrm{~m}^{3} / \mathrm{h}$ and a magnetic field of $7 \mathrm{~T}$.

It is possible to obtain the potential difference between electrodes, i.e., the electromotive force, by Ohm's law. Fig. 8 shows calculated values of electromotive force at magnetic fields of 6 and 7 T, comparing with the experimental and theoretical values. The theoretical values were calculated taking account of the average flow velocity (flow rate / cross section of helical flow) and the average external field of $90 \%$ of the maximum value. As can be seen in this figure, the calculated values agreed with the experimental values at a low flow rate but the experimental values were lower at a high flow rate.

\section{Recent Study on Hydraulic and Other Characteristics}

Effect of Flow Rectifier. To reduce the flow loss, and also to increase the electromotive force, experiments on the fundamental characteristics of the MHD generator were carried out using flow

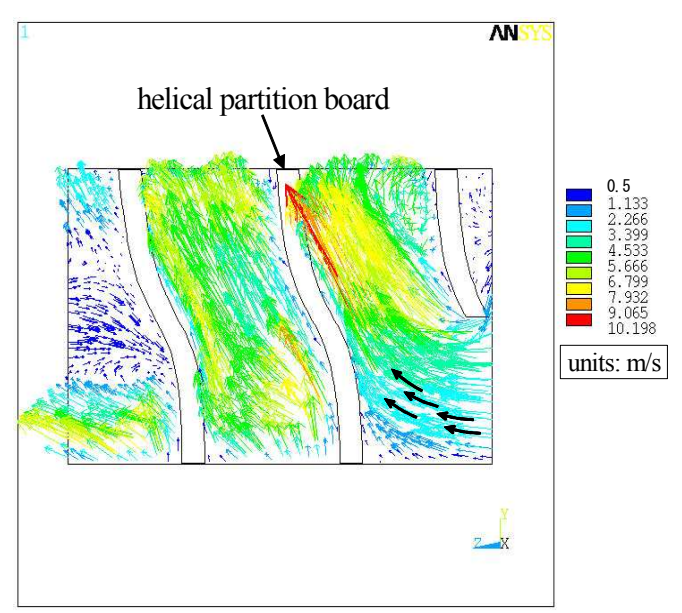

Fig. 6 Flow velocity distribution.

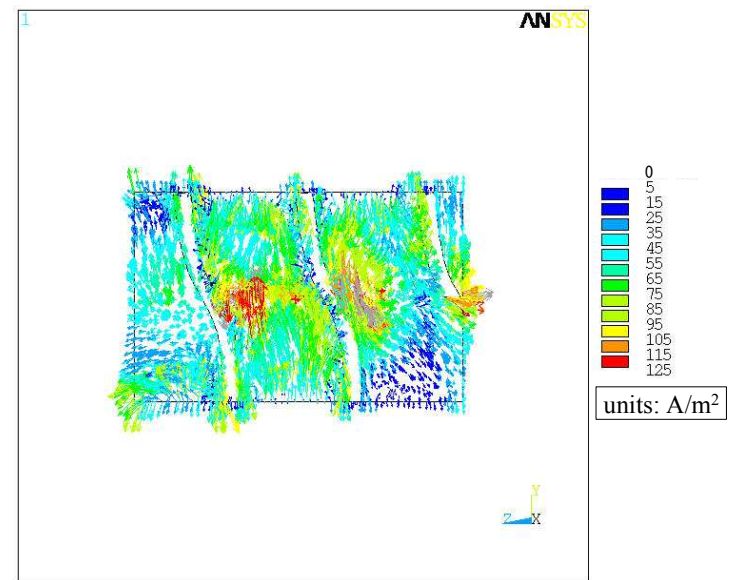

Fig. 7 An electric current density distribution. $(B=7 \mathrm{~T}, \Delta P=88.664 \mathrm{kPa})$ 


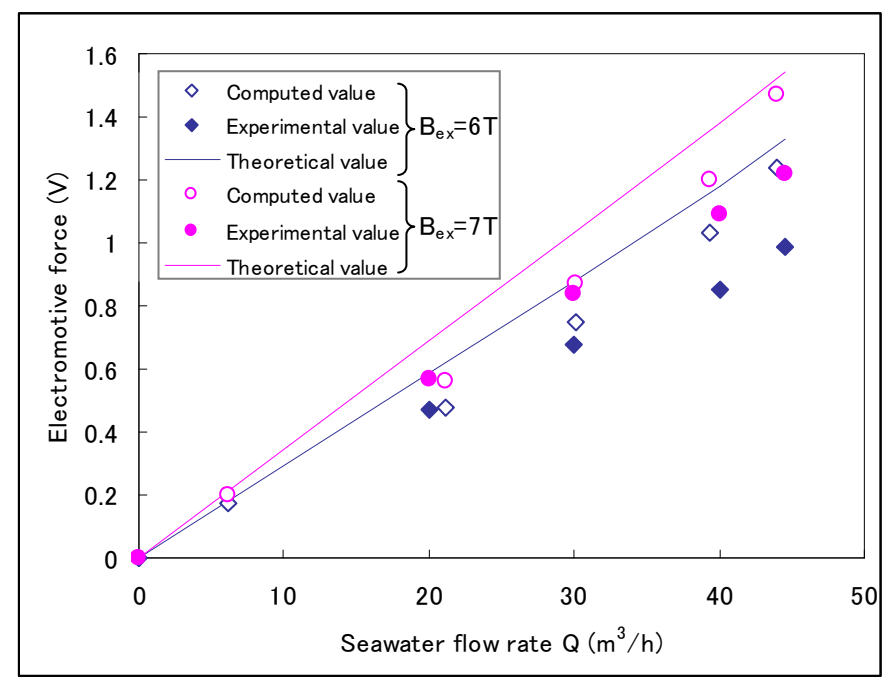

Fig. 8 Relationship between flow rate and electromotive force at $B=$ 6 and $7 \mathrm{~T}$.

rectifiers [5], which were installed in both the inlet and outlet of the generator. A photograph of a flow rectifier is shown in Fig. 9. The flow rectifier consists of a cylindrical tube $10 \mathrm{~mm}$ in thickness and six vanes $5 \mathrm{~mm}$ in thickness. The rectifier is made of polyvinyl-chloride-coated urethane foam and is $101.9 \mathrm{~mm}$ in diameter and $157.7 \mathrm{~mm}$ long. Fig. 10 shows the relationship between electromotive force and average flow velocity for the helical-type generator with flow rectifiers. The maximum value of the electromotive force was $1.35 \mathrm{~V}$, which was about $10 \%$ larger than that without flow rectifiers, as expected from the decrease in flow loss of about $20 \%$ with the flow rectifiers.

Effects of Configuration of Helical Wall. To construct a calculation model of flow loss, the effects of the rotation number $(3,5,7)$, pitch length $(30,37.5,45 \mathrm{~mm})$ and the inner diameter $(10,20$, $30 \mathrm{~mm}$ ) of the helical wall on flow loss were studied [6]. The experimental values for rotation numbers of 3 and 5 were in good agreement with the computed values at flow rates of $40 \mathrm{~m}^{3} / \mathrm{h}$ or less. The experimental values for a rotation number of 7 were in good agreement with the computed values at flow rates of $20 \mathrm{~m}^{3} / \mathrm{h}$ or less. However, the experimental values were about $17 \%$ larger than the computed values at flow rates from 30 to $40 \mathrm{~m}^{3} / \mathrm{h}$.

The experimental values for pitch lengths of $37.5 \mathrm{~mm}$ and $45 \mathrm{~mm}$ were in good agreement with the computed values at flow rates of 38 and $34 \mathrm{~m}^{3} / \mathrm{h}$ or less, respectively, whereas the experimental values for a pitch length of $30 \mathrm{~mm}$ were about 1.5-2 times larger than the computed values at flow rates from 10 to $28 \mathrm{~m}^{3} / \mathrm{h}$.

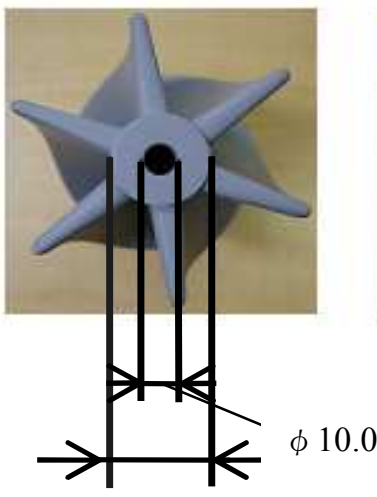

$\phi 30.4$
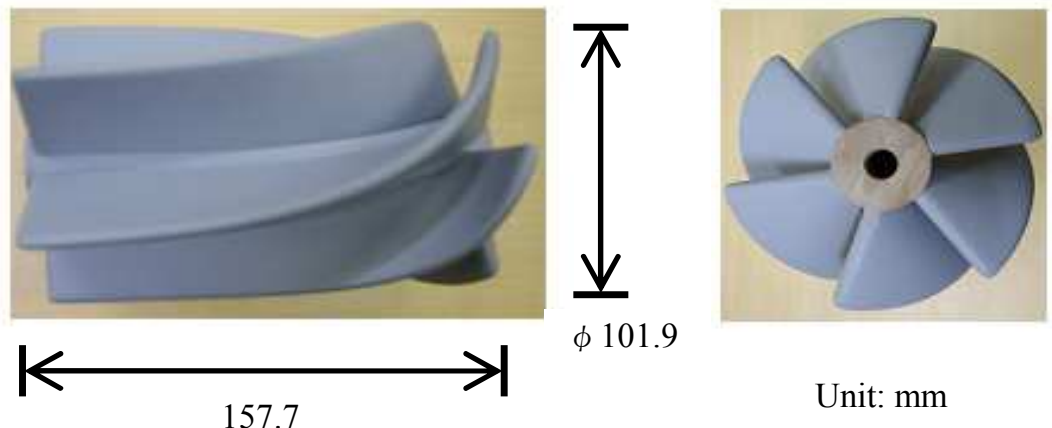

Unit: mm

Fig. 9 Photograph of a flow rectifier. 


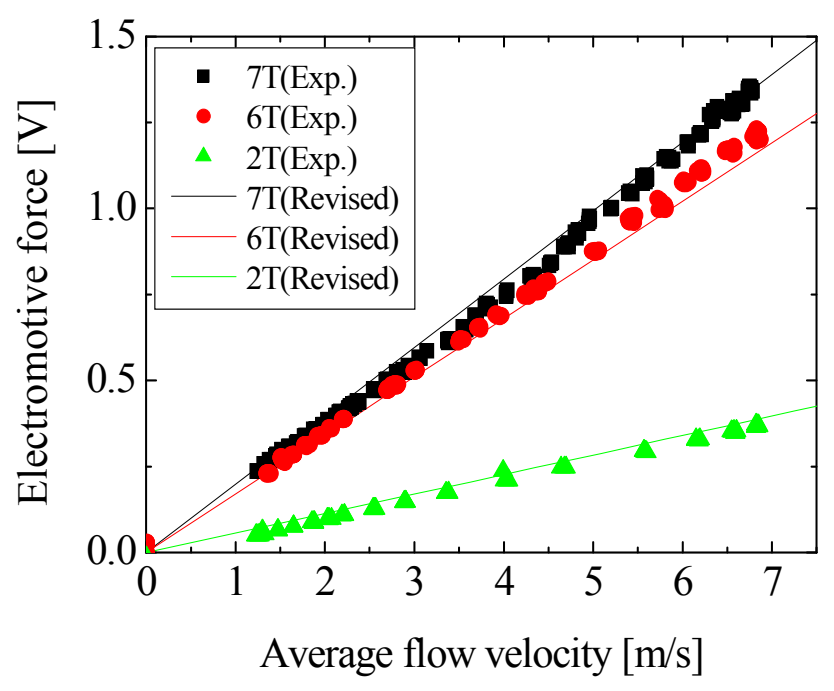

Fig. 10 Relationship between electromotive force and average flow velocity in comparison with revised theoretical values.

The experimental values for diameters of 10, 20 and $30 \mathrm{~mm}$ were in good agreement with the computed values at flow rates of 38,30 and $16 \mathrm{~m}^{3} / \mathrm{h}$ or less, respectively. However, for the diameter of $30 \mathrm{~mm}$ the computed values were about $20-23 \%$ larger than the experimental values at flow rates from 18 to $36 \mathrm{~m}^{3} / \mathrm{h}$.

Estimate of Performance. On the basis of a simple model, the performance of the helical-type generator was estimated using the experimental data for the hydraulic characteristics. The analysis of the output characteristics of the helical-type generator has been made continuously from the viewpoint of optimizing the configuration of the helical wall. We intend to carry out a test to evaluate the performance of the helical-type generator with the optimized configuration of the helical wall.

\section{Summary}

Experimental and computational results for the helical-type MHD power generator / hydrogen generator, which was designed as a new seawater MHD generator, have been discussed. On the basis of analytic results for the output characteristics, a helical-type generator with the optimized configuration of the helical wall will be constructed and tested in the near future.

\section{Acknowledgments}

The author would like to thank his research colleagues at Kobe University, Dr. T. Kiyoshi and Dr. X. Liu of National Institute for Materials Science, Japan. This work was supported by KAKENHI (18560767, 21360429), Hyogo Science and Technology Association, Kansai Research Foundation for Technology Promotion and NYK-Heyerdahl Projects, Japan.

\section{References}

[1] A. Iwata and Y. Saji: TEION KOGAKU (J. Cryo. Soc. Jpn.) Vol. 15 (1980) p. 317.

[2] K. Nishigaki, C. Sha, M. Takeda, Y. Peng, K. Zhou, A. Yang, D. Suyama, Q. J. Qing, L. Yan, T. Kiyoshi and H. Wada: Cryogenics Vol. 40 (2000) p. 353.

[3] M. Takeda, Y. Okuji, T. Akazawa, X. Liu and T. Kiyoshi: IEEE Trans. Appl. Supercond. Vol. 15 (2005) p. 2170.

[4] X. Liu, T. Kiyoshi and M. Takeda: Cryogenics Vol. 46 (2006) p. 362. 
[5] M. Takeda, Y. Iwamoto, T. Akazawa, K. A. Bui, T. Kida, X. Liu and T. Kiyoshi: J. Jpn. Inst. Marine Eng. Vol. 43 (2008) p. 130.

[6] K. A. Bui, M. Takeda, and T. Kiyoshi: submitted to J. Jpn. Inst. Marine Eng. 